\title{
THE PHYSICAL REALIZABILITY AND REALIZATION OF LINEAR PHASE SHIFT NETWORKS*
}

\author{
BY \\ PAUL M. CHIRLIAN \\ New York University
}

\begin{abstract}
Necessary and sufficient conditions for the physical realizability of linear phase shift networks are imposed upon the network transfer function. A technique is presented for approximating non-physically realizable transfer functions with those which are physically realizable. It is demonstrated that if the approximation is properly limited, the unit impulse response can be made non-negative for all values of time. These results are extended to networks whose transfer functions phase shift is the sum of two terms, one which varies linearly with frequency and a second which varies in discrete steps of $\pi$ radians.
\end{abstract}

Introduction. Investigations of the transient response of linear networks have led to the conclusion that a network transfer function whose phase shift is a linear function of frequency is often desirable. Of course, if such a network is to be built, it must be physically realizable. Necessary and sufficient conditions for this physical realizability have been obtained. The effect of these conditions on the transfer function can be readily visualized.

If a desired transfer function is not physically realizable, then it should be approximated by a transfer function which is physically realizable. An approximation of this type is presented, and it is shown that the accuracy of the approximation is improved as the magnitude of the slope of the phase shift (the network time delay) is increased. The unit impulse response of the approximating network can always be made a nonnegative function of time if the given transfer function is of finite bandwidth and the approximation is properly limited.

The following definition will be used throughout the body of the paper. The ratio, expressed as a complex function of frequency, of the steady state response of a network to its sinusoidal input will be called the transfer function. It will be written as $T(\omega)$ $\exp [j \theta(\omega)]$, where $T(\omega)$ is the amplitude function and $\theta(\omega)$ is the phase function. If $\theta(\omega)=-k \omega$, where $k$ is a real positive constant and $T(\omega)$ is a real positive function of $\omega$, then this is the transfer function of a linear phase shift network. If $T(\omega)$ takes on negative as well as positive values, then this will be called the transfer function of a stepped linear phase shift network. The term stepped is used since the negative values of $T(\omega)$ may be accounted for by adding discrete steps of $\pi$ radians to the phase function. The expression, generalized linear phase shift network, will be applied to networks which are either linear phase shift or stepped linear phase shift.

It shall be assumed that any amplitude functions considered here satisfy the Dirichlet conditions and, hence, possess a Fourier transform.

The physical realizability of generalized linear phase shift networks. If an arbitrary

\footnotetext{
*Received December 8, 1958. This paper is based upon a portion of a thesis which has been accepted by the faculty of the Graduate Division, College of Engineering, New York University, in partial fulfillment of the requirements for the degree of Doctor of Engineering Science.
} 
transfer function is assigned to a network, it is often found that the response of the network to a suddenly applied signal must appear before the signal is applied. Such a network is not physically realizable. In fact, the definition for physical realizability which shall be used in this paper is: A network is physically realizable if its response to a signal, applied at time $t=0$, is zero for all $t<0$. Hence, using the convolution integral, a necessary and sufficient condition for physical realizability is that the response of a network to a unit impulse applied at time $t=0$ is identically zero for all values of time less than zero. In order to convert this condition into one which can be interpreted physically, some properties of generalized linear phase shift networks will be discussed.

The unit impulse response $W(t)$ of a generalized linear phase shift network can be written as

$$
W(t)=\frac{1}{\pi} \int_{0}^{\infty} T(\omega) \cos \omega(t-k) d \omega .
$$

It can be seen that $W(t+k)$ is an even function of time. Hence, if a generalized linear phase shift network is to be physically realizable, it is necessary and sufficient that

$$
W(t+k)=0 \text { for } t<-k \text { and } t>k \text {. }
$$

It is of interest to note that the unit step response of a physically realizable generalized linear phase shift network is such that

$$
A(t+k)=\left\{\begin{array}{ll}
T(0), & t>k \\
0, & t<-k
\end{array} .\right.
$$

From the Fourier inversion relation we obtain

$$
T(\omega)=2 \int_{0}^{\infty} W(u+k) \cos \omega u d u,
$$

where $u=t-k$. Thus, we may state the following theorem:

Theorem 1. A necessary and sufficient condition that a generalized linear phase shift network, whose transfer function is $T(\omega) \exp (-j k \omega)$, be physically realizable is that $T(\omega)$ be expressible by the following Fourier transform:

$$
T(\omega)=\int_{0}^{k} H(u) \cos \omega u d u .
$$

Proof. The necessary condition is proven by assuming that $W(t)=0$ for $t<0$ and then applying Eq. (2) to Eq. (3) and then substituting $H(u)=2 W(u+k)$. The sufficiency condition is proven by hypothesizing Eq. (4), substituting $H(u)=2 W(u+k)$ and then comparing it with Eq. (3). Hence, $W(u+k)=0$ for $u>k$, but $W(u+k)$ is an even function of $u$. Therefore, $W(t)=0$ for $t<0$.

For an interpretation of Theorem 1 we can make use of the sampling theorem of Shannon [1]. From this theorem it can be seen that $T(\omega)$ is completely specified by a set of points spaced $\pi / k$ radians apart. Hence, increasing the magnitude of the phase slope, $k$, increases the accuracy of the approximation, in the sense that the function may be specified at more closely spaced points. It should be noted that the constant $k$ is also the delay time of the network. Thus, the maximum value of $k$ may be limited by the design constraints of the system. 
An approximation procedure. When an arbitrary generalized linear phase network transfer function $T_{1}(\omega)$ exp $(-j k \omega)$ is specified, often $T_{1}(\omega)$ does not satisfy the conditions imposed by Theorem 1. A technique is given here for approximating the given transfer function with a physically realizable transfer function, $T_{a}(\omega) \exp (-j k \omega)$. The form of the physically realizable approximate amplitude function, $T_{\bullet}(\omega)$, will depend upon the criterion of approximation used. In this case the Fourier criterion was used. That is, if $T_{1}(\omega)$ is of the form,

$$
T_{1}(\omega)=\int_{0}^{\infty} H_{1}(u) \cos \omega u d u,
$$

where

$$
H_{1}(u)=\frac{2}{\pi} \int_{0}^{\infty} T_{1}(\omega) \cos \omega u d \omega,
$$

then $T_{a}(\omega)$ will be defined as

$$
T_{a}(\omega)=\int_{0}^{k} H_{1}(u) \cos \omega u d u .
$$

An increase in $k$ will, of course, increase the accuracy of the approximation. Note that an increase in $k$ will not just introduce a constant time delay in this case because $T_{a}(\omega)$ will also vary.

Approximation procedures which guarantee a non-negative unit impluse response. It is often desirable for the unit impulse response of a network to be non-negative for all values of time (a monotonically increasing unit step response). If a transfer function of a linear phase shift network, $T_{1}(\omega) \exp (-j k \omega)$, which does not have a non-negative unit inpulse response, is specified, then often it is desirable to approximate $T_{1}(\omega)$ with a physically realizable approximate $T_{\bullet}(\omega)$, such that the resulting network has the desired non-negative unit impulse response. Procedures for obtaining such a $T_{\bullet}(\omega)$ will be presented. Two of these require that $T_{1}(\omega)$ be bandwidth limited. That is $T_{1}(\omega)=0$ if $\omega \geq \omega_{c}$, where $\omega_{c}$ is some specified angular frequency. In many instances such low pass characteristics are specified or closely approximate the specified characteristics.

In the following theorems it is assumed that the networks are generalized linear phase shift with a phase slope of magnitude $k$.

Theorem 2. If $T_{\Delta}(\omega)$ is a physically realizable approximate of some $T_{1}(\omega)$ where $T_{1}(\omega)=$ 0 if $\omega \geq \omega_{c}, T_{1}(\omega) \geq 0$ for $0 \leq \omega<\omega_{c}$, and $\omega_{c} k \leq \pi / 2$ then the unit impulse response will be non-negative for all values of time.

Proof. From Eq. (6) we obtain

$$
H_{1}(u)=\frac{2}{\pi} \int_{0}^{\omega c} T_{1}(\omega) \cos \omega u d \omega .
$$

For the range of integration, $T_{1}(\omega) \geq 0$ and $\cos \omega u \geq 0$, since $0 \leq \omega u \leq \omega_{c} k \leq \pi / 2$. Hence, $H_{1}(u) \geq 0$ for all $|u| \leq k$; but (from the proof of Theorem 1) $W(t)=H(t-k) / 2$ for $0 \leq t \leq 2 k$ and $W(t)=0$ for $t<0$ and $t>2 k$. Therefore $W(t) \geq 0$ for all $t$.

Thus, if the magnitude of the phase slope is equal to or less than $\pi /\left(2 \omega_{c}\right)$, the unit impulse response will be non-negative. It may be desired to obtain a larger phase slope magnitude, without altering the shape of the transient response (although time delay 
will be introduced). This may be accomplished by not increasing the upper limit of integration of Eq. (7) so that now $T_{a}(\omega)$ is given by

$$
T_{a}(\omega)=\int_{0}^{\pi /\left(2 \omega_{c}\right)} H_{1}(u) \cos \omega u d u .
$$

If, in addition to the requirements imposed in Theorem 2, the specified amplitude function $T_{1}(\omega)$ is a monotonically decreasing function of time, then the restrictions on $k$ imposed in Theorem 2, can be relaxed somewhat.

Theorem 3. If $T_{a}(\omega)$ is a physically realizable approximate of some $T_{1}(\omega)$ where $T_{1}(\omega)=$ 0 if $\omega \geq \omega_{c}, T_{1}(\omega) \geq 0$ for $0 \leq \omega<\omega_{c}, T_{1}(\omega)$ is a monotonically decreasing function of $\omega$, and $\omega_{\mathrm{c}} k \leq \pi$ then the unit impulse response will be non-negative for all values of time.

Proof. The proof follows that of Theorem 2 except the fact that $T_{1}(\omega)$ is a monotonically decreasing function of $\omega$ allows $\cos \omega u$ to take some negative values, provided $\omega u \leq \pi$.

Thus, a better approximation to $T_{1}(\omega)$ can be obtained when $T_{1}(\omega)$ is a monotonically decreasing function of $\omega$.

If the specified amplitude function $T_{1}(\omega)$ is such that

$$
\int_{0}^{\infty} T_{1}(\omega) \cos \omega u d \omega \geq 0 \text { for all } 0 \leq u \leq M,
$$

then any physically realizable approximate of $T_{1}(\omega)$ will result in a non-negative unit impulse response provided that $k \leq M$. An example of this is a $T_{1}(\omega)$ which decreases steadily to zero as $\omega$ approaches infinity and is convex downwards. The integral of Eq. (8) will be positive for all values of $u$, (see Titchmarsh [2]) Thus, any positive value of $k$ may be used.

As far as the problem of actually realizing these networks is concerned, it can be shown that these networks cannot be realized with a finite number of lumped linear elements. However, Corrington and Sonnenfeldt [3] and Kallman [4] have developed methods for realizing linear phase shift networks, using distributed elements. These techniques can be applied to generalized linear phase shift networks.

Conclusion. The definition of linear phase shift networks has been generalized to include networks with a transfer function $T(\omega) \exp (-j k \omega)$, where $T(\omega)$ is allowed to take on negative as well as positive values. If the network is to be physically realizable, then $T(\omega)$ must be expressible by the following Fourier integral:

$$
T^{\prime}(\omega)=\int_{0}^{k} H(u) \cos \omega u d u .
$$

One means of interpreting this result is that $T(\omega)$ can be specified at points which are no closer than $\pi / k$ radians per second apart.

A technique is presented for approximating any arbitrarily specified amplitude function $T_{1}(\omega)$ by a physically realizable amplitude function $T_{a}(\omega)$ which is given by

$$
T_{a}(\omega)=\int_{0}^{k} H_{1}(u) \cos \omega u d u,
$$

where

$$
H_{1}(u)=\frac{2}{\pi} \int_{0}^{\infty} T_{1}(\omega) \cos \omega u d u \quad u \leq k .
$$


If $T_{1}(\omega) \geq 0$ and is bandwidth limited, so that $T_{1}(\omega)=0$ if $\omega \geq \omega_{c}$, then the unit impulse response of the approximating network will be positive for all values of time, if $k \leq \pi /\left(2 \omega_{c}\right)$. If, in addition to the requirements just imposed upon $T_{1}(\omega)$, it is also a monotonically decreasing function of $\omega$ then the unit impulse response of the approximating network will be non-negative if $k \leq \pi / \omega_{c}$. Finally, it is shown that if

$$
\int_{0}^{\infty} T_{1}(\omega) \cos \omega u d \omega \geq 0 \text { for all } u \leq M,
$$

then, provided that $k \leq M$, any physically realizable approximate of $T_{1}(\omega)$ will have a non-negative unit impulse response. If the value of $k$ is increased without altering $T_{a}(\omega)$ then the transient response of the network will be delayed in time but its shape will not be altered. Thus, the value of $k$ discussed in this paragraph can be considered to be an upper bound on the upper limit of integration in Eq. (7) and a lower bound on the magnitude of the phase slope.

Acknowledgment. The author is indebted to Professor James H. Mulligan, Jr. for the many valuable suggestions and criticisms which were made during the course of this work.

The author also wishes to express his appreciation to Professor Armen H. Zemanian, Professor Charles F. Rehberg, and Professor Sheldon S. Chang for their many valuable comments.

\section{REFERENCES}

1. C. E. Shannon, Communication in the presence of noise, Proc. IRE 37, 10-21 (Jan. 1949)

2. E. C. Titchmarsh, Introduction to the theory of Fourier integrals, Oxford University Press, London, 1948 , p. 170

3. M. S. Corrington and R. W. Sonnenfeldt, Synthesis of constant time delay networks, R. C. A. Rev. 15, 163-186 (June 1954)

4. H. E. Kallman, Transversal filters, Proc. IRE. 28, 302-310 (July 1940) 